\title{
Towards a mechanism for instability in channel flow of highly shear-thinning viscoelastic fluids
}

\author{
Hugo A. Castillo and Helen J. Wilson \\ Mathematics Department, University College London \\ Gower Street, London WC1E 6BT, UK
}

June 8, 2017

\begin{abstract}
We consider the linear stability of channel flow of a shear-thinning viscoelastic fluid, replicating a instability recently discovered in experimental [1] and theoretical work [2]. We have extended the fluid model to allow for an inelastic shear-thinning stress component, and find that this additional contribution always has a stabilising influence on the instability. We conclude that, while shear-thinning is critical to the instability, the mechanism is primarily elastic.
\end{abstract}

\section{Introduction}

In recent experimental work, Bodiguel and coworkers [1] discovered a supercritical instability in channel flow of a viscoelastic shear-thinning fluid (a high molecular weight polymer solution). The steady rheometry of their fluid suggested that both the shear viscosity and the relaxation time of the fluid could be modelled with a power-law dependence on shear-rate.

This scenario was modelled theoretically by Wilson \& Loridan [2] using linear stability theory and a modified UCM model whose physical parameters (relaxation time and shear modulus) were allowed to depend instantaneously on the local shear rate. They had some success in reproducing the experimental observations, but no real insight into the mechanism of the instability.

It is clear that the instability is neither inertial (since it exists at zero Reynolds number) nor the well-known curved-streamline instability [3], since 
the streamlines are straight. The stability of shear-thinning flows without elasticity has been studied extensively $[4,5,6]$ and no instability has ever been found in the absence of inertia. A natural question to ask is whether the mechanism of this instability is truly elastic or principally a result of strong shear-thinning. To address this, we will augment the fluid model of [2] in the simplest way possible, and study the effect of reducing elasticity while maintaining the shear-thinning velocity profile.

\section{Model fluid}

We want a fluid which models the constitutive behaviour seen in experiments [1] using as few disposable parameters as possible, while also allowing some exploration of the importance of viscoelastic effects in triggering the observed instability; it is also useful to have a model which reduces to that used in [2] in some limit.

We choose to add just one term to the model used by Wilson \& Loridan [2]. We add a "solvent" contribution to the stress, which shear-thins at the same rate as the polymer contribution, in order to retain the simple power-law dependence of the shear viscosity. This will introduce only one new dimensionless parameter to the problem.

\subsection{Governing equations}

The set of governing equations for the incompressible shear-thinning fluid (in the absence of external body forces such as gravity) is:

\section{Continuity equation}

$$
\underline{\nabla} \cdot \underline{u}=0
$$

\section{Momentum equation}

$$
\rho\left(\frac{\partial \underline{u}}{\partial t}+\underline{u} \cdot \underline{\nabla u}\right)=\underline{\nabla} \cdot \underline{\underline{\sigma}}
$$

\section{Constitutive model}

$$
\begin{gathered}
\underline{\underline{\sigma}}=-p \underline{\underline{I}}+\mu(\dot{\gamma}) \underline{\underline{D}}+G(\dot{\gamma}) \underline{\underline{A}} \\
\underline{\underline{A}}=-\frac{1}{\lambda(\dot{\gamma})}(\underline{\underline{A}}-\underline{\underline{I}})
\end{gathered}
$$

in which the upper-convected derivative is defined as

$$
\underline{\underline{A}}=\frac{\partial \underline{\underline{A}}}{\partial t}+u \cdot \underline{\underline{A}} \underline{\underline{A}}-\underline{\underline{A}} \cdot(\underline{\nabla} \underline{u})-(\underline{\nabla} \underline{u})^{\top} \cdot \underline{\underline{A}} .
$$


Here $\underline{u}$ is the fluid velocity, $\rho$ its density, and $p$ is the pressure. The tensor $\underline{\underline{A}}$ is the conformation tensor, describing the stretch in the polymer chains; $\underline{\underline{I}}$ is the identity tensor, $\underline{\underline{\sigma}}$ is the total stress tensor, and we have introduced a symmetric flow gradient tensor

$$
\underline{\underline{D}}=\underline{\nabla} \underline{u}+\underline{\nabla} \underline{u}^{\top} .
$$

The scalar functions $G$ (the shear modulus), $\lambda$ (the relaxation time) and $\mu$ (the viscosity of the solvent) are all empirical functions of the shear-rate $\dot{\gamma}$, defined as

$$
\dot{\gamma}=\sqrt{\frac{1}{2} \underline{\underline{D}}: \underline{\underline{D}}} .
$$

The only difference between this model and that used in [2] is the addition of the solvent term $\mu(\dot{\gamma}) \underline{\underline{D}}$ in equation (3). This additional term will give us a shear-thinning modification of the Oldroyd-B model, which will allow us to separate elastic from viscous effects.

\section{$2.2 \quad$ Rheometry}

We now consider the behaviour of our fluid in a simple shear flow, $\underline{u}=\dot{\gamma} y \underline{e}_{x}$ with $\dot{\gamma}>0$, using cartesian coordinates. The stress tensor for our system is:

$$
\underline{\underline{\sigma}}=\left(\begin{array}{cc}
-P_{0}+G\left(1+2 \lambda^{2} \dot{\gamma}^{2}\right) & (\mu+\lambda G) \dot{\gamma} \\
(\mu+\lambda G) \dot{\gamma} & -P_{0}+G
\end{array}\right) .
$$

The viscometric functions accessible in experiment are:

$$
\sigma_{12}=(\mu+G \lambda) \dot{\gamma}, \quad N_{1}=\sigma_{11}-\sigma_{22}=2 G \lambda^{2} \dot{\gamma}^{2},
$$

in which the parameters $\mu, G$ and $\lambda$ depend on the shear-rate $\dot{\gamma}$. In the original experiments of [1], both these viscometric functions were observed to have a power-law dependence on $\dot{\gamma}$, so we follow [2] in defining

$$
G(\dot{\gamma})=G_{M} \dot{\gamma}^{m-n} \quad \lambda(\dot{\gamma})=K_{M} \dot{\gamma}^{n-1}
$$

and, to keep the shear stress $\sigma_{12}$ a simple power-law, we introduce

$$
\mu(\dot{\gamma})=\mu_{0} \dot{\gamma}^{m-1} .
$$

The one new parameter of this model, $\mu_{0}$, is the solvent-viscosity coefficient. The resulting rheological response of our model fluid is

$$
\begin{gathered}
\sigma_{12}=\left(\mu_{0}+G_{M} K_{M}\right) \dot{\gamma}^{m} \\
\frac{\sigma_{11}-\sigma_{22}}{2 \sigma_{12}}=\left(\frac{G_{M} K_{M}^{2}}{\mu_{0}+G_{M} K_{M}}\right) \dot{\gamma}^{n} .
\end{gathered}
$$


The fluid rheology of a viscoelastic shear-thinning fluid reported in [1] is:

$$
\sigma_{12}=3.73 \dot{\gamma}^{0.21} \mathrm{~Pa} \quad \frac{\sigma_{11}-\sigma_{22}}{2 \sigma_{12}}=3.63 \dot{\gamma}^{0.43} .
$$

Matching these experimental fits to our model (equations (12-13)), and taking $\mu_{0}$ in units of $\mathrm{Pas}^{0.21}$, we obtain:

$$
\begin{gathered}
m=0.21 \quad n=0.43 \\
G_{M}=\left(\frac{3.73-\mu_{0}}{K_{M}}\right) \operatorname{Pas}^{0.22} \quad K_{M}=3.63\left(1+\frac{\mu_{0}}{3.73-\mu_{0}}\right) \mathrm{s}^{0.43}
\end{gathered}
$$

The power-law parameters $m$ and $n$ are unchanged from their values in [2], but now the parameters $K_{M}$ and $G_{M}$ are only determined once a value has been chosen for $\mu_{0}$ (which must lie between 0 and $3.73 \mathrm{~Pa} \mathrm{~s}^{0.21}$ ). As $\mu_{0}$ increases, $K_{M}$ also increases but $G_{M}$ decreases, as shown in figure 1 . If $\mu_{0}=0$ we regain the values used in [2], namely $G_{M}=3.73 \mathrm{Pas}^{0.22}$ and $K_{M}=3.63 \mathrm{~s}^{0.43}$.

\subsection{Steady channel flow}

As in [2], we consider a two-dimensional channel flow of infinite extent in the $x$-direction, half height $L$ (in the $y$-direction) and driven by a constant pressure gradient $\mathcal{P}$ in the $x$-direction. We assume a steady, unidirectional flow profile $\underline{u}=U(y) \underline{e}_{x}$ satisfying a no-slip condit ion at $y= \pm L$. The following velocity profile is obtained:

$$
U(y)=\left(\frac{\mathcal{P}}{\mu_{0}+G_{M} K_{M}}\right)^{1 / m}\left(\frac{m}{m+1}\right)\left[L^{(m+1) / m}-|y|^{(m+1) / m}\right],
$$

from which an important flow measure is the centreline velocity

$$
U_{0}=U(0)=\left(\frac{\mathcal{P}}{\mu_{0}+G_{M} K_{M}}\right)^{1 / m}\left(\frac{m}{m+1}\right) L^{(m+1) / m} .
$$

The only difference here with respect to the velocity profile in [2] is the addition of the solvent viscosity $\mu_{0}$, that appears in the denominator of the first term of the equation (17).

\subsection{Dimensionless form of the governing equations}

In order to reduce our parameter space, we derive dimensionless forms of our equations. We scale lengths with $L$, we use the average shear rate $U_{0} L^{-1}$ to scale times, and scale stresses with a typical shear stress, which is $\left(\mu_{0}+\right.$ $\left.G_{M} K_{M}\right)\left(U_{0} / L\right)^{m}$. In terms of dimensionless variables, the governing equations become:

$$
\underline{\nabla} \cdot \underline{u}=0
$$




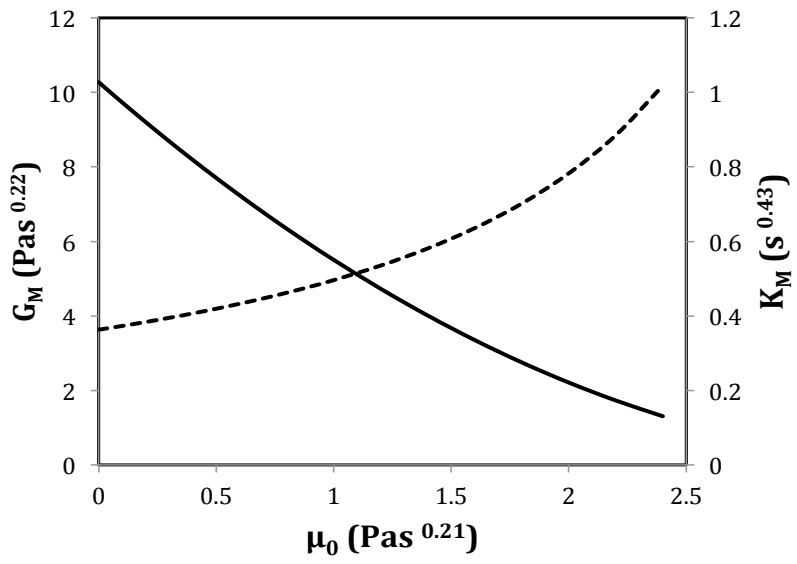

Figure 1: Plot of the required behaviour of the parameters $G_{M}$ (solid line) and $K_{M}$ (dashed line) as the solvent coefficient $\mu_{0}$ varies, in order to correctly replicate the experimental data from [1]. Curves specified by equation (16). 


$$
\begin{gathered}
\operatorname{Re}\left(\frac{\partial \underline{u}}{\partial t}+\underline{u} \cdot \underline{\nabla u}\right)=\underline{\nabla} \cdot \underline{\underline{\sigma}} \\
\underline{\underline{\sigma}}=-p \underline{\underline{I}}+\left(\frac{\beta}{1+\beta}\right) C \underline{\underline{D}}+\left(\frac{1}{1+\beta}\right) \frac{C}{\mathcal{W}} \underline{\underline{A}} \\
\underline{\underline{A}}=-\frac{1}{\mathcal{W}}(\underline{\underline{A}}-\underline{\underline{I}}) \\
C=\dot{\gamma}^{m-1} \quad W=W \dot{\gamma}^{n-1} \quad W=K_{M}\left(\frac{U_{0}}{L}\right)^{n}
\end{gathered}
$$

The functions $C$ and $\mathcal{W}$ are the dimensionless viscometric functions, and there are three dimensionless physical constants: $W, R e$ and $\beta . W$ is the purely elastic Weissenberg number; the Reynolds number $R e$ is expressed as:

$$
R e=\frac{\rho U_{0} L}{(1+\beta) G_{M} K_{M}\left(U_{0} / L\right)^{m-1}} ;
$$

and we have introduced a new dimensionless parameter, $\beta$, which is the $r e$ tardation parameter: the ratio between the solvent viscosity and the polymer viscosity:

$$
\beta=\frac{\mu_{0}}{G_{M} K_{M}} .
$$

The domain of values of $\beta$ is $[0, \infty)$, with the limit $\beta=0$ corresponding to the earlier work of Wilson \& Loridan [2]. We will mostly concern ourselves with the weak effects of solvent, $0<\beta<1$, but will consider some cases of larger $\beta$ (corresponding to solvent-dominated flow).

\section{Stability calculation}

\subsection{Base state}

In order to study the stability of the flow, we first need a steady solution. In dimensionless form, the velocity profile, shear rate and viscometric functions become:

$$
\begin{gathered}
U=1-|y|^{\frac{m+1}{m}} \quad y \in[-1,1] \\
\dot{\gamma}_{0}=\left|U^{\prime}\right|=\frac{m+1}{m}|y|^{1 / m} \\
C_{0}=\dot{\gamma}_{0}^{m-1}=\left[\frac{m+1}{m}\right]^{m-1}|y|^{(m-1) / m} \\
\mathcal{W}_{0}=W \dot{\gamma}_{0}^{n-1}=W\left[\frac{m+1}{m}\right]^{n-1}|y|^{(n-1) / m} .
\end{gathered}
$$

Equations (26-29) are exactly the same as equations (15-17) of [2]). The pressure, conformation and stress tensor are:

$$
P_{0}=P_{\infty}+\frac{1}{1+\beta} \frac{C_{0}}{\mathcal{W}_{0}}+\mathcal{P} x
$$




$$
\begin{gathered}
\underline{\underline{A}}=\left(\begin{array}{ll}
A_{11} & A_{12} \\
A_{21} & A_{22}
\end{array}\right)=\left(\begin{array}{cc}
1+2 W^{2}[\mathcal{P} y]^{2 n / m} & -W[\mathcal{P} y]^{n / m} \\
-W[\mathcal{P} y]^{n / m} & 1
\end{array}\right) \\
\underline{\underline{\sigma}}=\left(\begin{array}{cc}
\Sigma_{11} & \Sigma_{12} \\
\Sigma_{21} & \Sigma_{22}
\end{array}\right)=\left(\begin{array}{cc}
-P_{\infty}+\mathcal{P} x+\frac{2 W}{(1+\beta)}[\mathcal{P} y]^{\frac{m+n}{m}} & -\mathcal{P} y \\
-\mathcal{P} y & -P_{\infty}+\mathcal{P} x,
\end{array}\right)
\end{gathered}
$$

and the first normal stress difference is:

$$
N_{1}=\Sigma_{11}-\Sigma_{22}=\frac{2 W}{(1+\beta)}[\mathcal{P} y]^{\frac{m+n}{m}}
$$

where $P_{\infty}$ is a baseline pressure and $\mathcal{P}=[(m+1) / m]^{m}$ is now the dimensionless pressure gradient (its value was dictated by the normalization of the velocity profile).

These differ from the previous work [2] only in the normal stress component $\Sigma_{11}$. This term is reduced here by a factor of $(1+\beta)$ because of the presence of the solvent (which does not contribute to the normal stress difference but does contribute to the shear stress we use as our stress scale). In the limit $\beta \rightarrow \infty$, the solvent dominates and we have a simple power-law fluid: a Generalised Newtonian Fluid having no viscoelastic effects.

We should also note here an error in equation (20) of [2]: the term $\Sigma_{11}$ is written there as $-P_{\infty}+\mathcal{P} x+2 W \mathcal{P}^{n} y^{(m+n) / m}$. The correct form is $-P_{\infty}+\mathcal{P} x+$ $2 W[\mathcal{P} y]^{(m+n) / m}$.

\subsection{Perturbation flow}

A spatially periodic perturbation is added to the base flow, so all quantities are modified by an infinitesimally small change:

$$
\begin{gathered}
\underline{u}=(U+u \varepsilon, v \varepsilon) \\
\dot{\gamma}=\dot{\gamma}_{0}+\dot{\gamma}_{1} \varepsilon \\
C=C_{0}+c \varepsilon \\
\mathcal{W}=\mathcal{W}_{0}+w \varepsilon \\
\underline{\underline{A}}=\left(\begin{array}{ll}
A_{11}+a_{11} \varepsilon & A_{12}+a_{12} \varepsilon \\
A_{21}+a_{21} \varepsilon & A_{22}+a_{22} \varepsilon
\end{array}\right) \\
\underline{\underline{\sigma}}=\left(\begin{array}{ll}
\Sigma_{11}+\sigma_{11} \varepsilon & \Sigma_{12}+\sigma_{12} \varepsilon \\
\Sigma_{21}+\sigma_{21} \varepsilon & \Sigma_{22}+\sigma_{22} \varepsilon
\end{array}\right)
\end{gathered}
$$

We consider a single Fourier mode with the form:

$$
\varepsilon=\epsilon \exp [i k x-i \omega t]
$$


where $k$ is the wavenumber and $\omega$ is the frequency. Applying Fourier modes to the conservation equation, we obtain:

$$
i k u+\frac{d v}{d y}=0
$$

Let us introduce the streamfunction $\psi$, setting:

$$
u=\frac{d \psi}{d y} \quad v=-i k \psi .
$$

We also introduce the notation $D$ to denote differentiation with respect to $y$. Substituting the perturbed forms into the governing equations, and discarding terms of order $\varepsilon^{2}$, we obtain a linearised system of equations:

$$
\begin{gathered}
\operatorname{Re}(-i \omega D \psi-i k \psi D U+i k U D \psi)=i k \sigma_{11}+D \sigma_{12} \\
\operatorname{Re}\left(-k \omega \psi+k^{2} U \psi\right)=i k \sigma_{12}+D \sigma_{22} \\
\underline{\underline{\sigma}}=-p \underline{\underline{I}}+\left(\frac{\beta}{1+\beta}\right)[\underline{\underline{\underline{D}}}+C \underline{\underline{d}}]+\frac{1}{(1+\beta)}\left[\frac{C}{\mathcal{W}} \underline{\underline{a}}+\left(\frac{c}{\mathcal{W}}-\frac{C w}{\mathcal{W}^{2}}\right) \underline{\underline{A}}\right] \\
\underline{\underline{D}}_{0}=\left(\begin{array}{cc}
0 & D U \\
D U & 0
\end{array}\right) \quad \underline{\underline{d}}=\left(\begin{array}{cc}
2 i k D \psi & D^{2} \psi+k^{2} \psi \\
D^{2} \psi+k^{2} \psi & -2 i k D \psi
\end{array}\right) \\
\left(-i \omega+i k U+\mathcal{W}^{-1}\right) a_{11}=i k \psi D A_{11}+2 a_{12} D U+2 A_{11} i k D \psi \\
+2 A_{12} D^{2} \psi+\frac{w}{\mathcal{W}^{2}}\left(A_{11}-1\right) \\
\left(-i \omega+i k U+\mathcal{W}^{-1}\right) a_{12}=i k \psi D A_{12}+a_{22} D U+A_{11} k^{2} \psi+ \\
D^{2} \psi+\frac{w}{\mathcal{W}^{2}} A_{12} \\
\left(-i \omega+i k U+\mathcal{W}^{-1}\right) a_{22}=2 A_{12} k^{2} \psi-2 i k D \psi
\end{gathered}
$$

In order to determine the perturbation to the shear-rate, we limit our domain to the upper half of the channel $0 \leq y \leq 1$, in which $D U<0$ and thus $\dot{\gamma}_{0}=-D U$. This allows us to calculate $\dot{\gamma}_{1}$ using the definition:

$$
\dot{\gamma}_{0}+\dot{\gamma}_{1} \varepsilon=\left(\frac{1}{2}\left[\underline{\underline{D}}_{0}+\varepsilon \underline{\underline{d}]}\right]:\left[\underline{\underline{D}}_{0}+\varepsilon \underline{\underline{d}}\right]\right)^{1 / 2}+O\left(\varepsilon^{2}\right) .
$$

The perturbation shear rate and the perturbed form of the viscometric functions are:

$$
\begin{aligned}
\dot{\gamma}_{1} & =-\left(D^{2}+k^{2}\right) \psi, \\
c & =(m-1) \dot{\gamma}_{0}^{m-2} \dot{\gamma}_{1}, \\
w & =W(n-1) \dot{\gamma}_{0}^{n-2} \dot{\gamma}_{1} .
\end{aligned}
$$




\subsection{Boundary conditions and centreline singularity}

We have a coupled system of equations (43-52). Those equations can be combined and the resulting equation is a fourth-order ODE in $\psi$ dependent on $y$. The boundary conditions are conditions of no flow on the boundaries:

$$
\psi=D \psi=0 \text { at } y= \pm 1 .
$$

The system is governed by six dimensionless parameters: the five introduced in [2] (the indices $m$ and $n$, the Weissenberg and Reynolds numbers, and the wavenumber $k$ ) along with the new parameter $\beta$. We solve the ODE using the shooting method of Ho \& Denn [7].

As described in previous works $[8,2]$, we face a common problem as result of using power-law models, which happens at the centreline $y=0$ : the viscometric functions become singular at that point, which is unphysical. However, if we limit ourselves to varicose perturbations, for which $\psi$ is an odd function of $y$, then the value of the perturbed shear rate $\dot{\gamma}_{1}$ will be zero at the centreline, allowing us to draw some conclusions without having to further complicate the model. This means our new boundary conditions become

$$
\psi=D \psi=0 \text { at } y=1, \quad \psi=D^{2} \psi=0 \text { at } y=0 .
$$

Note that we are not claiming that the varicose modes are the most unstable - indeed, the experimental observations of [1] suggest that sinuous modes may be more dangerous. Rather, the limitations of the power-law model restrict the modes to which linear stability theory can be applied. In future we intend to study models which do not suffer from the power-law singularity, and thereby answer the question of the relative stability of sinuous and varicose modes.

\section{Results}

\subsection{Effect of solvent on growth rate}

\subsubsection{Fluid to match experiments}

For a direct comparison with the experimental instability seen in [1], we fix $m=0.20$ and $n=0.40$. The physical parameters $G_{M}$ and $K_{M}$ must be chosen to match equation (16) for a given value of $\mu_{0}$; however, in the dimensionless equation set the variation of these physical quantities is all captured within the dimensionless parameter $\beta$.

The case $\beta=0$ was studied by Wilson \& Loridan [2], who found instability in the absence of inertia for Weissenberg numbers above 1.8; increasing the flow rate to $W=2$ they found (their figure $6(\mathrm{a})$ ) a broad peak in the plot of growth 
rate against wavenumber, with the most unstable mode at $k=4.18$ but instability being seen over the large range $2.2<k<24$. Here we choose an indicative wavenumber $k=3$ (giving a wavelength, $2 \pi / k$, of the same magnitude as the channel) and investigate the effect of increasing $\beta$ from zero: that is, increasing the solvent contribution and correspondingly decreasing the viscoelastic contribution to the stress.

The results are shown in figure 2. We see that the growth rate of the instability is reduced monotonically by the introduction of solvent, and the flow becomes stable to perturbations of this wavelength for $\beta>0.0695$. In the complex plane, we see that as the unstable mode becomes stable, its advection rate $\Re(\omega)$ increases, suggesting that it localises closer to the centre of the channel.

\subsubsection{Fluid having constant shear modulus}

Our second case study fluid is a highly shear-thinning fluid whose shear modulus $G$ is constant: we set $m=n=0.2$ to have

$$
C=\dot{\gamma}^{-0.8} \quad \mathcal{W}=W \dot{\gamma}^{-0.8}
$$

This fluid was shown by Wilson \& Rallison [8] to be unstable to waves of wavenumber $k \approx 3$ at a Weissenberg number $W=2$ without inertia, though the growth rate is lower than for the fluid discussed in section 4.1.1 above.

We see the stability behaviour of this fluid as function of the retardation parameter $\beta$ in figure 3: as before, although the initial instability observed at $\beta=0$ remains present at small values of retardation parameter, the growth rate decreases and reaches stability at $\beta>0.0135$.

\subsubsection{Critical retardation parameter value}

In the previous sections, we analysed the stability of two fluids to perturbations of a fixed wavenumber $k=3$. It is more useful to determine the critical value of retardation parameter for each fluid: the value of $\beta$ above which the fluid is stable to perturbations of all wavelengths. In this section we fix a value of the relaxation time power-law coefficient $m=0.2$, along with Weissenberge number $W=2$ and zero Reynolds number $R e=0$, and for a range of values of $n$, we have calculated the critical $\beta$ above which the flow is unconditionally linearly stable. The results are shown in figure 4 .

We see that if $n>0.5235$ (fluids whose shear modulus is strongly shearthinning) or $n<0.1846$ (shear-thickening modulus), the flow is stable for any value of $\beta$ : i.e. these fluids are stable even without the addition of solvent. As $n$ decreases from a starting point $n=0.5234$ (where a tiny addition of solvent is sufficient to stabilise the flow) we see an increase in the critical retardation parameter. The curve reaches its maximum value at $n=0.42$ : for this rheology, 
a)

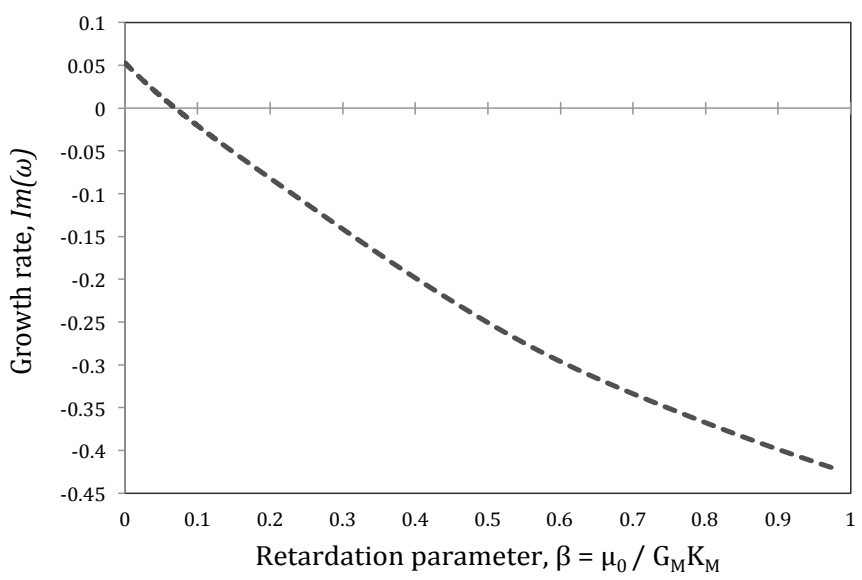

b)

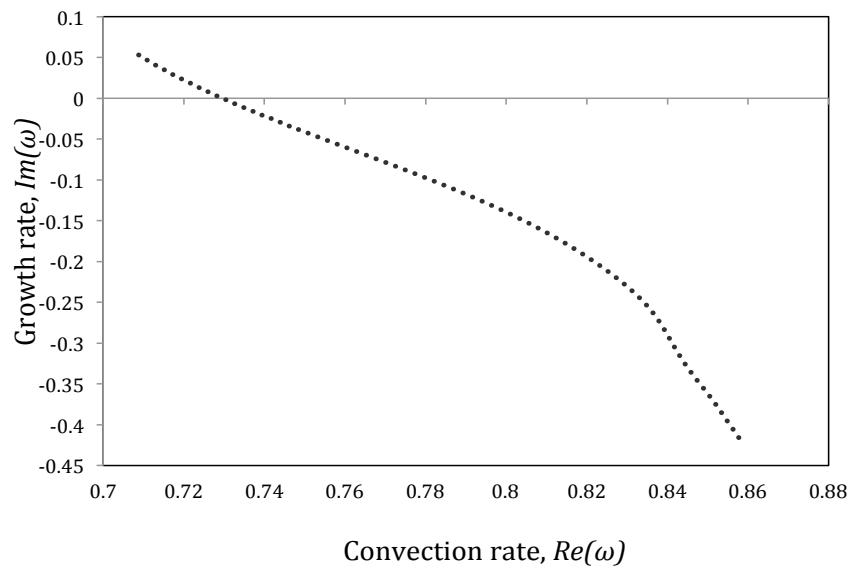

Figure 2: Variation of the instability with the retardation parameter $\beta$ at $m=$ $0.2, n=0.4, W=2, R e=0$ and $k=3$. (a) Plot of growth rate $\Im(\omega)$ against $\beta$. (b) Behaviour of the eigenvalue $\omega$ in the complex plane. 
a)

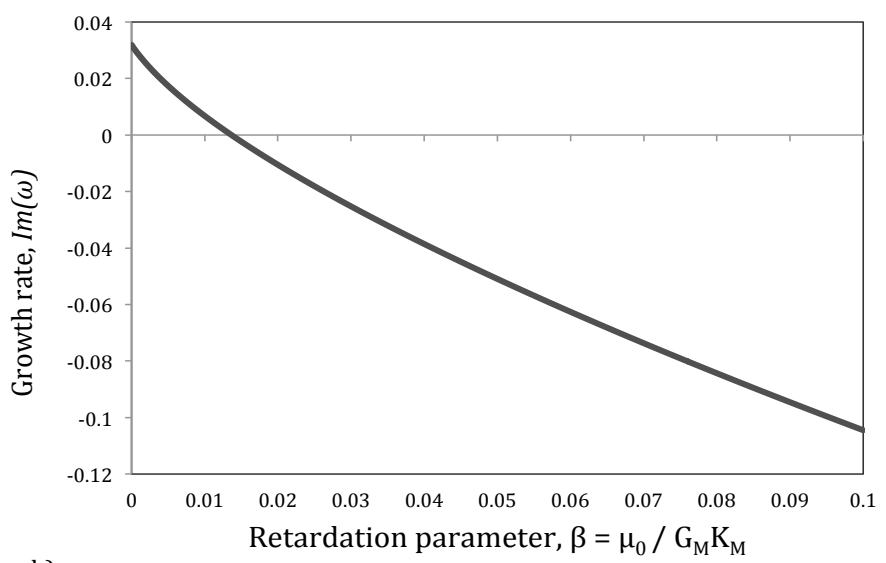

b)

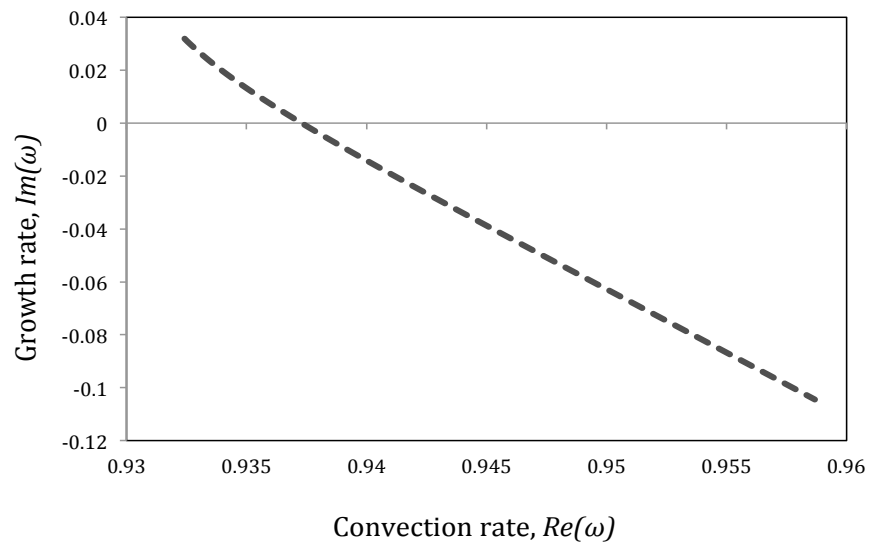

Figure 3: Variation of the instability with the retardation parameter $\beta$ for a fluid with constant shear modulus constant: $m=n=0.2, W=2, k=3$ and $R e=0$. (a) Plot of growth rate $\Im(\omega)$ against $\beta$. (b) Behaviour of the eigenvalue $\omega$ in the complex plane. 
a value of $\beta=0.0804$ would be required to completely stabilise the flow. This fluid is not very different from that used in the experiments of [2].

A local minimum is observed at $n=0.246$. After this point, the plot rises again and a second maximum is located at $n=0.216$ (a very weakly shearthinning shear modulus). Below this second maximum, the critical retardation parameter value decreases monotonically until at the point $n \approx 0.1846$ (a slightly shear-thickening modulus) the instability is lost even when there is no solvent viscosity.

Note that both the fluids discussed in the previous sections have the same value of $m=0.2$, which means they exhibit the same flow profile; the constantmodulus fluid $(n=0.2)$, which is only marginally more stable than the experimentallymatched fluid $(n=0.4)$ in the absence of solvent, becomes stable much sooner as solvent viscosity is added to both fluids.

From figures 2-4 it is clearly seen that, at least for these test cases, the first effect of adding a solvent that shear thins at the same rate than the polymer is to stabilise the flow. This suggests that viscoelasticity, rather than shear-thinning alone, is a critical component of the mechanism of instability.

\subsection{Critical Weissenberg number}

In the previous section we showed that adding solvent can stabilise flows at a fixed Weissenberg number. However, for a more systematic picture of the instability, and particularly of onset, it is of interest to know the critical Weissenberg number: the dimensionless flow rate below which the flow is stable to perturbations of all wavenumbers.

One such calculation was carried out by Wilson \& Loridan [2]: there was no solvent $(\beta=0)$, inertial terms were neglected $(R e=0)$, the power-law coefficient $m=0.2$ was fixed, and a range of values of the timescale powerlaw $n$ was considered. They found that the lowest critical Weissenberg number (the most unstable fluid at this value of $m$ ) was located at $n=0.2$, with a second local minimum around $n=0.4$, close to the fluid used in experiments [1]. These results are reproduced in figure 5, along with the corresponding curves for nonzero retardation parameter.

We see that, in all cases, the critical Weissenberg number increases with increasing $\beta$ : that is, as we had already postulated, the presence of inelastic solvent is stabilising. At $\beta=0$ (the case presented in [2]) there is a sharp minimum in the curve at $n=0.2$ (for which the shear modulus is constant, $n=m$ ) and a broader minimum around $n=0.4$ (a shear-thinning modulus similar to the fluid used in [1]). The constant-modulus case $n=0.2$ becomes unstable at a lower critical Weissenberg number than any other fluid. The critical Weissenberg number increases markedly for large or small $n$.

As $\beta$ is increased, the sharp minimum first becomes broader and more stable 


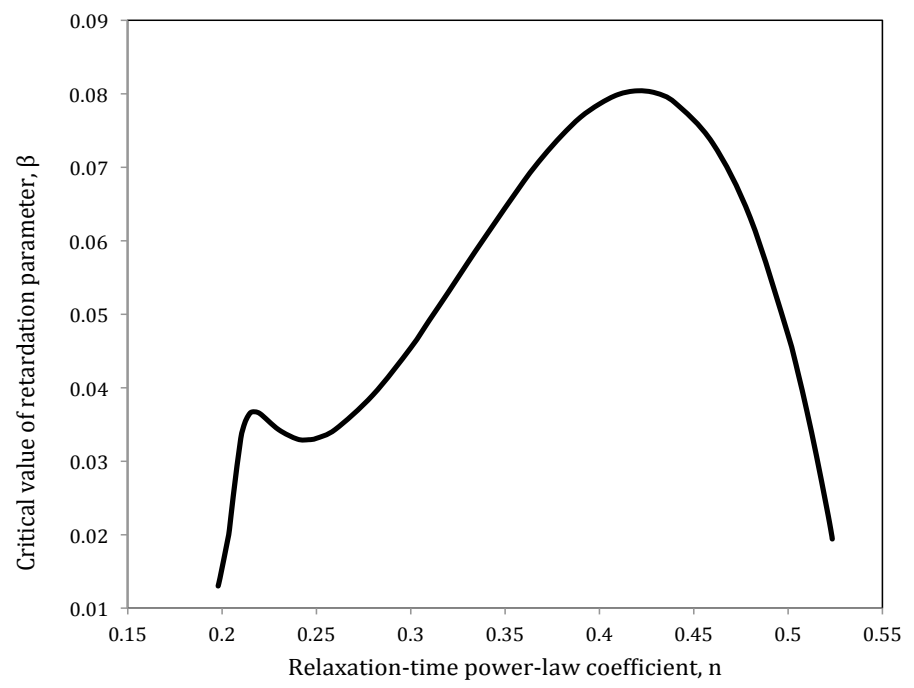

Figure 4: Critical retardation parameter value $\beta$ plotted against relaxation time power-law $n$; the other parameters are fixed as $m=0.2, R e=0, W=2$. 
a)

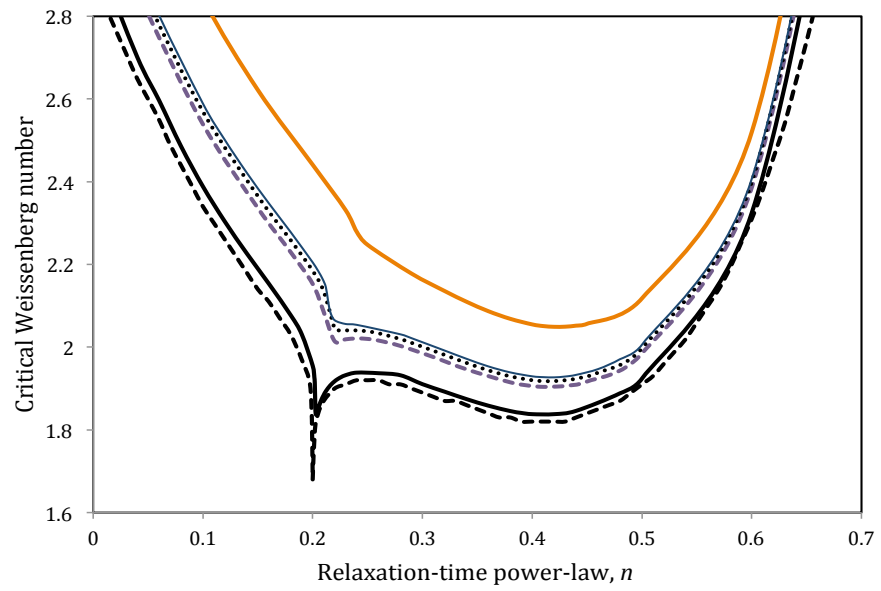

b)

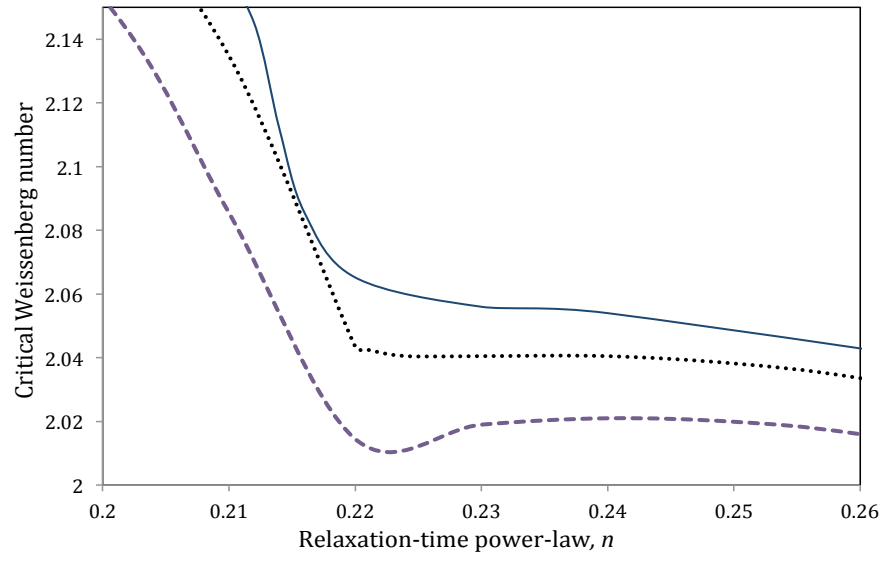

Figure 5: Critical Weissenberg number plotted against the relaxation time power-law, $n$, for different values of the retardation parameter $\beta$, in the absence of inertia $(R e=0)$. We have fixed $m=0.2$. (a) Increasing from bottom: $\beta=0,0.01,0.04,0.046,0.05,0.1$; (b) the disappearance of the local minimum near $n=0.2$ : (from bottom) $\beta=0.04,0.046,0.05$. 
(this happens very quickly; even a very small solvent contribution $\beta=0.01$ is enough to make this fluid more stable than the shear-thinning modulus case $n \approx 0.4$ ), moves to slightly larger values of $n$, and then disappears completely at $\beta=0.046$ (shown in figure 5(c)). Beyond this value, the dominant instability is the broad minimum which started at $n \approx 0.4$; this also shifts slightly to higher $n$.

As $\beta$ increases further, there are no more exciting developments in the critical Weissenberg number curve, which has one simple minimum. The flow becomes more stable (the critical Weissenberg number increases), and the most dangerous fluid (at $m=0.2$ ) is found at increasingly larger values of $n$.

The results shown in figure 5 are consistent with those we saw in figure 4: the local minimum located at $n=0.4$ in the plot of critical Weissenberg number stabilises relatively slowly with the addition of solvent, so requires a larger value of $\beta$ to stabilise the flow. On the other hand, for the lowest $W_{\text {crit }}$ seen at $m=n=0.2$ when $\beta=0$, the effect of solvent is more dramatic and a much lower value of $\beta$ is sufficient to banish the instability.

\subsubsection{Case study fluid: Experimental matching}

In this section we take the fluid rheology measured for the fluid used in the experiments of [1], that is, $m=0.2, n=0.4$, and examine the evolution of the critical Weissenberg number with changing the retardation parameter $\beta$. We have seen already that $W_{\text {crit }}$ increases with increasing $\beta$ when $\beta$ is small; in figure 6 we plot it against $\beta$ for a wider range.

At values of $\beta<0.1$, the growth of $W_{\text {crit }}$ with $\beta$ is approximately linear $\left(W_{\text {crit }} \approx 1.82+2.3 \beta\right)$, but for larger $\beta$ the growth is faster and by a value of $\beta=1$ (when the total shear viscosity has equal components from the solvent and elastic contributions) we have $W_{\text {crit }}=8.25$.

At much higher values of $\beta$, the required flow rate for instability becomes very large; and extrapolating from the data shown in figure 6 , we expect that in the limit $\beta \rightarrow 0$, for which there is no elastic contribution to the stress, the flow will be unconditionally stable at all Weissenberg numbers.

\subsubsection{Dependence of critical Weissenberg number on shear stress power law, $m$}

We have seen in previous sections that the effect of adding solvent viscosity is purely stabilising: that is, the mechanism of this instability must be in some part elastic. However, shear-thinning must also be a critical component of the instability, since the non-shear-thinning case $m=n=1$, the Oldroyd-B fluid, is known to be linearly stable in this flow at $R e=0[7,9]$.

The following question then arises: how strongly does shear-thinning affect 


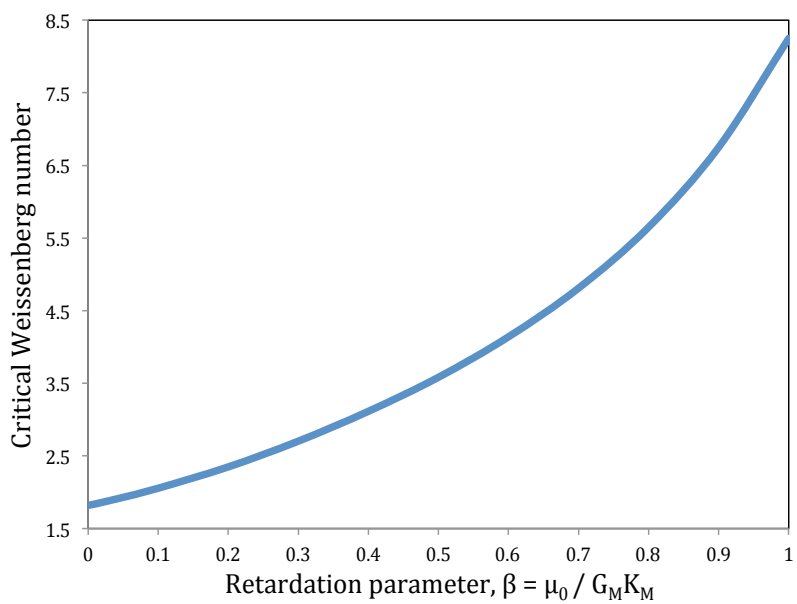

Figure 6: Plot of the critical Weissenberg number against the retardation parameter $\beta$ for an exemplar fluid defined by $m=0.2, n=0.4$. 
this instability? To address this, we now study the dependence of the critical Weissenberg number on the power-law coefficient $m$, which governs the level of shear-thinning in the shear viscosity.

In this section we set $\beta=R e=0$; we vary both the remaining fluid parameters $m$ and $n$, and we determine the critical Weissenberg number $W_{\text {crit }}$ for each flow: the Weissenberg number below which the flow is linearly stable to perturbations of all wavenumbers, $k$.

In figure $7(\mathrm{a})$ and (b) we show the results: a plot of $W_{\text {crit }}$ against $n$ for three different values of the power-law coefficient, $m=0.20,0.19$ and 0.18 . The shape of the three curves is similar: $W_{\text {crit }}$ is large when the relaxation time power-law $n$ is either large or small, and the most unstable scenario (lower $W_{\text {crit }}$ ) occurs at $m=n$. The case $m=0.18$ is the most unstable of the three.

The less sharp minimum in the $W_{\text {crit }}$ curve, which for the case $\beta=0$ is seen at $n=0.4$, remains located at the same value of timescale $n$ for the cases $m=0.19$ and $m=0.18$, but with different values of $W_{\text {crit }}$ for each.

If we now add solvent viscosity, the general behaviour of the curves of $W_{\text {crit }}$ against $n$ will not be different from that observed for $m=0.2$ (figure 5): the flow will be more stable as the retardation parameter increases, the lowest point seen at $m=n$ when $\beta=0$ will no longer be the most unstable scenario, and the lowest point of the global curves will be located in a zone of weakly-shear thinning modulus near $n=0.4$. The main difference between the three cases ( $m=0.20, m=0.19$ and $m=0.18$ ) is that the most unstable curves will be found when the power-law coefficient $m$ is equal to 0.18 .

From these observations, we might be tempted to conclude that decreasing the power-law coefficient $m$ would produce fluids that are less and less stable; we might guess that the most unstable scenarios would be found in strongly shear-thinning fluids $(m \rightarrow 0)$. However, this is not the case: in figure $7(\mathrm{c})$ we give a plot of growth rate against power-law coefficient $m$, having fixed the other parameters $n=0.4, \beta=R e=0, W=2, k=3$. We chose these parameters to match one of the most unstable scenarios in figure $7(\mathrm{a})$.

The instability only exists in the small region $0.1<m<0.205$; the strongest instability occurs at $m \approx 0.18$, while very strongly shear-thinning fluids having $m \rightarrow 0$ are fully stable, which is consistent with the observations originally made by Wilson \& Loridan [2]. In that limit, we also observed that the flow is stable for any value of Weissenberg number (and therefore, no critical Weissenberg number was found).

\section{Conclusions}

We have investigated the stability of channel flow of a strongly shear-thinning viscoelastic fluid, modelled to imitate the experiments of [1]. Extended the 


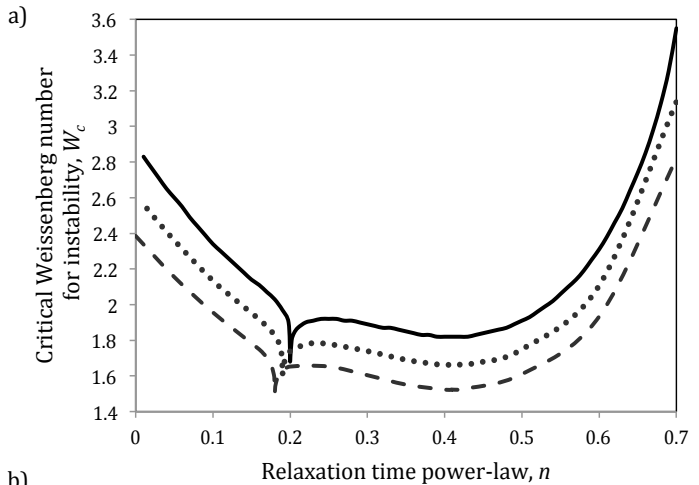

b)
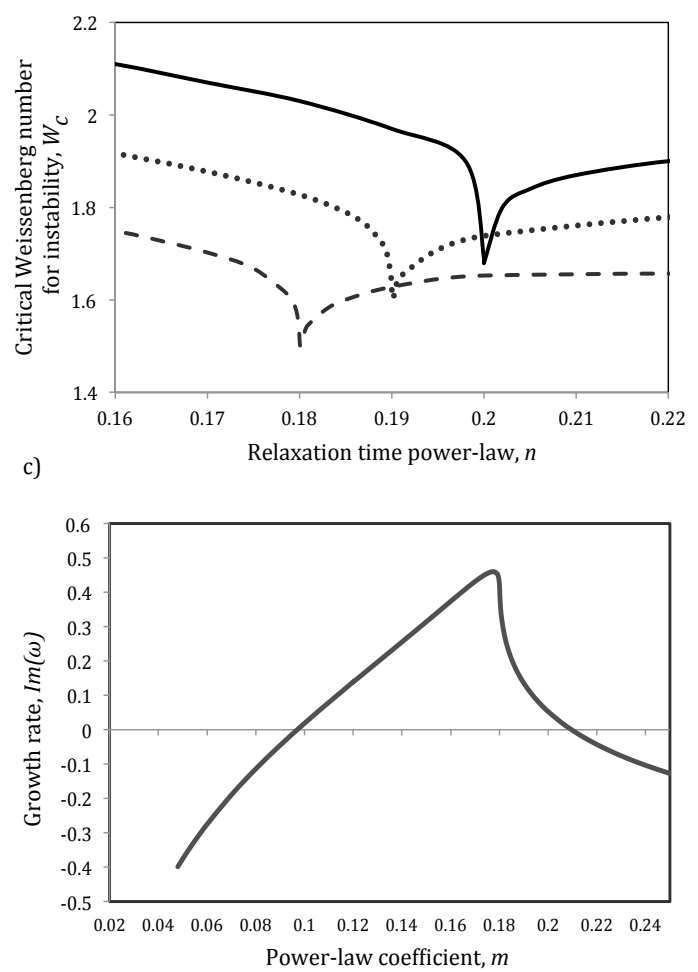

Figure 7: The effect of shear-thinning when $\beta=R e=0$. (a) Plot of the critical Weissenberg number $W_{\text {crit }}$ against relaxation time power-law $n$, for three different values of the shear-stress power law $m$. Solid line: $m=0.20$ (identical to figure 5 of [2]; dotted line: $m=0.19$; dashed line: $m=0.18$. (b) Small region of plot (a). (c) Dependence of the growth rate on $m$ for specific values of the other parameters: $n=0.4, W=2, k=3, R e=0, \beta=0$. 
existing model of [2], we introduced a non-Newtonian solvent contribution to the fluid stress, which does not change the shear-rate dependence of the fluid's shear viscosity but reduces the effect of viscoelasticity.

We introduced a new parameter: the retardation parameter, $\beta$, defined as the ratio the solvent to polymer contributions to the shear viscosity of the fluid. We found out that for a wide range of values of the other key parameters in the problem, the addition of solvent (the increase of $\beta$ ) has a stabilising effect on the flow. Since the solvent effectively reduces the size of the elastic terms in the system, we can conclude that elasticity is a key factor in the mechanism of this instability.

Two specific fluid models stand out in our analysis: the case in which the shear modulus is constant, originally studied by Wilson \& Rallison [8]; and a shear-thinning modulus fluid close to that used in the experiments of Bodiguel et al. [1]. These fluids are both extreme in the sense that any fluid whose physical parameters are very close to one of these specific cases will be marginally more stable than our specific fluid. In the absence of solvent, the constant-shearmodulus fluid is the more unstable of the two; but it is stabilised more quickly than the shear-thinning modulus case, which rapidly becomes the dominant instability for finite values of $\beta$.

We also assessed the dependence of the instability on the degree of shearthinning in the shear stress, described by the power-law $m$; it was observed that there is a critical level of shear-thinning for which the instability is strongest; for either weak shear-thinning $m \rightarrow 1$ or very strong shear-thinning $m \rightarrow 0$, the flow is unconditionally linearly stable. Even strong elastic shear-thinning is not the only component of this instability: some complex coupling between shear and normal stress effects is at work.

The instability cannot be caused by inertial terms, as it appears even when we fix a Reynolds number of zero. Nor is it exclusively caused by inelastic nonNewtonian effects (shear-thinning), as the addition of a shear-thinning solvent term is strongly stabilising. We conclude that the instability is of purely elastic nature; and we observe that its strongest effects may be observed for a fluid whose shear viscosity thins very strongly (with a power-law coefficient $m \approx 0.2$ ) and whose elastic modulus is weakly shear-thinning $\left(G \sim \dot{\gamma}^{-0.2}\right)$.

Acknowledgment: This work was supported financially by the National Council of Science and Technology of Mexico (CONACyT).

\section{References}

[1] H. Bodiguel, A. Beaumont, A. Machado, L. Martinie, H. Kellay and A. Colin, Flow enhancement due to elastic turbulence in channel flows of shearthinning fluids, PRL 114 (2015) 028302. 
[2] H. J. Wilson and V. Loridan, Linear instability of a highly shear-thinning fluid in channel flow, Journal of Non-Newtonian Fluid Mechanics 223 (2015), 200-208.

[3] P. Pakdel and G. H. McKinley. Elastic instability and curved streamlines. Physical Review Letters, 77 (1996) 2459-2462.

[4] C. Nouar, A. Bottaro and J. Brancher, Delaying transition to turbulence in channel flow: revisiting the stability of shear-thinning fluids, Journal of Fluid Mechanics 592 (2007), 177-194

[5] C. Nouar and I. Frigaard, Stability of plane Couette - Poiseuille flow of shear-thinning fluid, Physics of Fluids 21 (2009), 064104.

[6] A. Chekila, C. Nouar, E. Plaut and A. Nemdili, Subcritical bifurcation of shear-thinning plane Poiseuille flows, Journal of Fluid Mechanics 686 (2011), 272-298

[7] T. C. Ho and M. M. Denn, Stability of plane Poiseuille flow of a highly elastic liquid, Journal of Non-Newtonian Fluid Mechanics 3 (1978), 179.

[8] H. J. Wilson and J. M. Rallison, Instability of channel flow of a shearthinning White-Metzner fluid, Journal of Non-Newtonian Fluid Mechanics 87 (1999), 75-96.

[9] R. Sureshkumar and A. N. Beris, Linear stability analysis of viscoelastic Poiseuille flow using an Arnoldi-based orthogonalization algorithm, Journal of Non-Newtonian Fluid Mechanics 56 (1995), 151. 